cities, such as sufferers from the acquired immune deficiency syndrome and the homeless, who also need more medical attention. To reduce the work patients would have to be turned away and directed to go elsewhere. Such socialist control is against the spirit of the formation of the National Health Service, in which, with more and more difficulty, we try to keep our doors open to those who wish to come from near or far to the hospital. We are therefore weary of the continuous sniping that suggests that the large and thereby expensive workload in London is due to doctors practically asking patients to come here.

If patients want and need health care they should be able to obtain it. I can assure Ms Smith that there is morbidity here in London as well as in the north and am sad that an editor of the $B M F$ supports ideas that are devisive between health districts.

Rayne Institute,

St Thomas's Hospital,

London SE1 7EH

\section{AIDS related consultations in a general practice}

SIR,-Except for those general practitioners in contact with a large population of intravenous drug users, ${ }^{1}$ there have been few descriptions of the size and nature of the problem caused by the acquired immune deficiency syndrome (AIDS) as it presents to a typical general practice. From 1 November 1986 to 30 April 1987 we therefore recorded all AIDS related consultations in our practice of five doctors, one practice nurse, and 11700 patients.

An AIDS related consultation was defined as any consultation by: $(a)$ someone with AIDS or an AIDS related illness or known to be positive for human immunodeficiency virus (HIV); $(b)$ any member of a high risk group presenting with anxieties about AIDS; $(c)$ any member of a low risk group-the worried well-seeking advice; or $(d)$ any patient asking for an HIV test for employment or insurance purposes. After each consultation the reason for the person's attendance, the risk group he or she belonged to, and what actions were undertaken were recorded.

From November 1986 to April 1987 there were 60 AIDS related consultations-33 new and 27 return. This represented about 1 in every 230 surgery consultations. All new consultations were initiated by the patient. Thirty patients presented initially to the doctor while the remaining three approached the practice nurse.

The largest group presenting (20) were the worried well, 11 were in high risk groups, and two requested the blood test for employment reasons. Only one seropositive patient, a haemophiliac, consulted during the study period. All high risk groups were represented, with the biggest number being intravenous drug users. ${ }^{5}$

Most of the patients (20) presented with worries about AIDS, and 12 of them asked for a blood test, four because they had an illness perceived to be AIDS (all in the worried well group) and three because of fears about future conception. Most consultations (24) resulted in simple advice and reassurance; in 17 cases the patients were counselled about the blood test, in 13 about safer sex,

AIDS related consultations from November 1986 to April 1987

\begin{tabular}{lcccccc}
\hline Month: & Nov & Dec & Jan & Feb & Mar & Apr \\
\hline New consultations & 4 & 2 & 7 & 6 & 11 & 3 \\
Return consultations & 1 & 7 & 5 & 6 & 8 & 0
\end{tabular}

and in six about drug addiction. One drug addict was given a supply of sterile needles and syringes. At least nine people were given a blood test, seven by the general practitioner and two by the sexually transmitted disease clinic. All were negative.

Our findings confirm anecdotal evidence that general practitioners are seeing an appreciable number of people, from both high and low risk groups, with worries about AIDS. The number of consultations rose in line with the second government AIDS advertising campaign and extensive media coverage. The decline in consultations seemed to correlate with the decline in publicity and is similar to that observed elsewhere. ${ }^{2}$

The largest group presenting were the worried well-those who perceive themselves to be at high risk despite an absence of risk factors. ${ }^{3}$ Our patients covered a wide range, from those with minor worries to a few with more serious psychological problems. The largest high risk group presenting were intravenous drug users. This is not surprising as a recent study has shown that general practitioners are the main medical agency in contact with drug users. ${ }^{4}$ This has important implications for general practitioners, who are well placed to deliver preventive measures to this elusive group.

I thank my partners and practice nurse for recording their consultations and for helpful discussion.

\section{MoRRIS GALLAGHER}

South Shields,

Tyne and Wear NE33 3PD

1 Robertson JR, Bucknall ABV, Welsby DD, et al. Epidemic of AIDS related virus (HTLV LLL/LAV) infection among intravenous drug users. BrMed f 1986;292:527-9.

2 Anderson R, Underhill G, Kerry C, et al. AIDS publicity campaign. Lancet 1987;i:1429-30.

3 Miller D. The worried well. In: Miller D, Weber J, Green J, eds. The management of AIDS patients. London: MacMillan, 1986. 4 Glanz A, Taylor C. Findings of a national survey of the role of general practitioners in the treatment of opiate misuse: exten of contact with opiate misusers. Br Med F 1986;293:427-30.

\section{How to take a sabbatical}

SIR,-It was heartenung to read Dr Ian Tait's article (12 September, p 644) persuading doctors to take a sabbatical from general practice to bring new life and awareness to their work on their return.

One way to find an awareness of how others live in different cultures-and tackle what seem to us almost insurmountable problems-is for doctors to offer their services for a period of two years to Voluntary Service Overseas (VSO). This is a personal and professional way to help build long term plans for health services in developing countries. Working with VSO includes developing primary health care facilities, managing and organising local hospitals and clinics, and training medical technicians.

VSO always needs to recruit more doctors. At the moment there are jobs for district medical officers in a number of places including Zambia and Papua New Guinea. A leprologist is requested in Nigeria, as well as a junior doctor to work with patients with leprosy in another hospital in Nigeria. A surgeon is needed for Malawi, a radiologist and pathologist for Belize, a doctor to organise rural dispensaries in Uganda. The posts are exciting and varied and a complete change from the surgery or the hospital at home.

VSO takes people aged between 20 and 65 who can commit themselves for at least two years and are willing to work for a local wage. It does not take dependants, but in special cases arrangements can be made for a partner to accompany the VSO worker. Return air fares, medical insurance, national insurance, equipment grants, and accommodation are provided. Health authorities will often give leave of absence if a volunteer requests it. For further information please contact the inquiries unit, VSO, 9.Belgrave Square, London SW1X 8PW.

vso,

Margaret LeguM

London SW1X 8PW

Benefits of effective hospital services for the elderly

SIR,-Dr John Mitchell and his colleagues (17 October; p 980) have given an impressive account of their unit and have clearly shown the advantages of an age related admission policy.

They state that other authors, including myself, have expressed despair at the ability of geriatric medicine departments to provide a hospital service with a reduced number of beds. This was certainly not my intention. What I stated was that it is impossible to provide an acute admission service for all elderly patients in a district and provide the same number of hospital based continuing care beds if the total number of beds is cut drastically. In 1981 my unit had 217 beds, of which 100 were designated as continuing care. Today it has 132 beds, through which I treated over 1600 patients last year, the district having introduced an over 75 age related admission policy early in 1986 . I doub whether even the most efficient geriatricians could treat this number of patients if 100 of their 132 beds were occupied by continuing care patients. Clearly I have been able to accommodate this number of patients only by reducing the number for which we provide continuing care. Unfortunately, in my district the savings achieved by closing geriatric beds have been used to breach the revenue gap rather than being diverted to the community unit to pay for the cost of caring for patients at home who in former days would have remained in hospital based continuing care beds. If the funds had been diverted I expect that I would have been able to cope with even fewer beds Overall therefore my experience mirrors that of the Whipps Cross team.

Peter Crome

George Stamp Unit,

Orpington Hospital,

Orpington, Kent BR6 9JU

1 Coid J, Crome P. Bed blocking in Bromley. Br Med f 1986;292: 1253-6.

Computer based system for assessing dietary intake

SIR,-We agree completely with Ms Jennifer Keogh and colleagues (26 September, p 785) that if accurate assessments of dietary intake are required weighed assessments are necessary. Indeed, weighed assessments were used as the gold standard in our study to validate the computer based system. However, it is rarely possible to assess dietary intake in the clinical setting using weighed assessments and it is inconceivable that weighed assessments could be applied routinely. Our system was designed to provide a routine measure of dietary intake which would be more convenient and accurate than the interview methods based on 24 hour recall used at present.

The system for assessing dietary intake described by Dr A S McIntyre and colleagues ( 26 September p 785) has a number of disadvantages in comparison with our own. Firstly, the dietary assessment is carried out using a questionnaire from which the information is extrapolated indirectly whereas with our system the food intake is entered directly into the computer by the subject. Secondly, their 